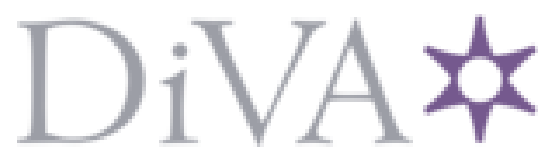

http://www.diva-portal.org

\title{
Postprint
}

This is the accepted version of a paper published in Chemical Physics. This paper has been peerreviewed but does not include the final publisher proof-corrections or journal pagination.

Citation for the original published paper (version of record):

Zagorodskikh, S., Zhaunerchyk, V., Mucke, M., Eland, J H., Squibb, R J. et al. (2015)

Single-photon double and triple ionization of acetaldehyde (ethanal) studied by multi-electron coincidence spectroscopy.

Chemical Physics, 463: 159-168

http://dx.doi.org/10.1016/j.chemphys.2015.10.006

Access to the published version may require subscription.

N.B. When citing this work, cite the original published paper.

Permanent link to this version:

http://urn.kb.se/resolve?urn=urn:nbn:se:uu:diva-270927 


\section{Single-photon double and triple ionization of acetaldehyde (ethanal) studied by multi-electron coincidence spectroscopy}

S. Zagorodskikh, ${ }^{1,2}$ V. Zhaunerchyk, ${ }^{2,1}$ M. Mucke, ${ }^{1}$ J. H. D. Eland, ${ }^{3,2,1}$ R. J. Squibb, ${ }^{2,1}$

L. Karlsson, ${ }^{1}$ P. Linusson, ${ }^{4}$ and R. Feifel ${ }^{2,1, a)}$

1) Department of Physics and Astronomy, Uppsala University, Box 516, SE-751 20 Uppsala, Sweden

${ }^{2)}$ Department of Physics, University of Gothenburg, Origovägen 6B, SE-412 96 Gothenburg, Sweden

3) Department of Chemistry, Physical and Theoretical Chemistry Laboratory, Oxford University, South Parks Road, Oxford OX1 3QZ, United Kingdom

4) Department of Physics, Stockholm University, AlbaNova University Center, SE-106 91 Stockholm, Sweden

(Dated: 21 September 2015)

Single-photon multiple ionization processes of acetaldehyde (ethanal) have been experimentally investigated by utilizing a multi-particle coincidence technique based on the time-of-flight magnetic bottle principle, in combination with either a synchrotron radiation source or a pulsed helium discharge lamp. The processes investigated include double and triple ionization in the valence region as well as single and double Auger decay of core-ionized acetaldehyde. The latter are studied site-selectively for chemically different carbon core vacancies, scrutinizing early theoretical predictions specifically made for the case of acetaldehyde. Moreover, Auger processes in shake-up and core-valence ionized states are investigated. In the cases where the processes involve simultaneous emission of two electrons, the distributions of the energy sharing are presented, emphasizing either the knock-out or shake-off mechanism.

\footnotetext{
a)Electronic mail: raimund.feifel@physics.gu.se
} 


\section{INTRODUCTION}

Experimental single photon double ionization studies of atoms and molecules were carried out already at the end of the 1960s in form of Auger electron spectroscopy ${ }^{1-3}$ with primary focus on diatomic and triatomic compounds. Subsequently, they were followed by experimental $^{4-7}$ and theoretical ${ }^{8,9}$ investigations of the Auger decay of polyatomic molecules. The majority of these investigations were performed using electrostatic hemispherical electron energy analyzers which provide relatively high resolution, but which detect electrons only within comparatively small kinetic energy ranges and with limited capability to obtain information on the inherent energy correlation of the emitted electrons.

Double photoionization electron coincidence spectroscopy based on a magnetic bottle introduced by Eland et al. ${ }^{10}$ in 2003 opened up the possibility to determine the correlation of electrons with a wide range of kinetic energies. To begin with, this method was used to study valence double ionization of atoms and molecules ${ }^{10-19}$ using a pulsed He lamp, and was subsequently applied to studies of core-valence double photoionization of atoms and molecules ${ }^{20-26}$ utilizing synchrotron radiation sources. Both processes are examples of double ionization, where the two electrons emitted may share the excess energy provided by the absorbed photon arbitrarily, in contrast to fixed electron kinetic energies typically observed in cases of single core shell ionization followed by normal Auger decay. This method was successfully expanded to the study of valence triple ionization ${ }^{27,28}$, where the excess photon energy is shared between three emitted electrons, and to the case of double Auger decay ${ }^{29,30}$ of a single core vacancy.

Acetaldehyde is an organic compound denoted chemically as $\mathrm{CH}_{3} \mathrm{CHO}$. Its electron configuration in the neutral ground state can be expressed as:

$$
\text { core }\left(4 a^{\prime}\right)^{2}\left(5 a^{\prime}\right)^{2}\left(6 a^{\prime}\right)^{2}\left(7 a^{\prime}\right)^{2}\left(1 a^{\prime \prime}\right)^{2}\left(8 a^{\prime}\right)^{2}\left(9 a^{\prime}\right)^{2}\left(2 a^{\prime \prime}\right)^{2}\left(10 a^{\prime}\right)^{2}
$$

where the three core orbitals 1a', 2a' and 3a' are localized on the oxygen and two different carbon atoms. Those orbitals are essentially atomic-like ${ }^{31}$ and are therefore referred to as $\mathrm{O} 1 s$ and $\mathrm{C} 1 s$ orbitals in what follows.

From previous work (see e.g. Refs. 32 and 33) it is known that compounds with two core orbitals localized on atoms of the same element, but in different chemical environments, may demonstrate different properties in the Auger processes depending on the core orbital 
involved. Acetaldehyde is interesting in this respect as it contains one carbon atom in a methylic group and another carbon atom in a formylic group. This offers the possibility of site-specific investigations of photoionization processes which involve the core orbitals, such as single and double Auger decay. The single ionization valence photoelectron spectrum of acetaldehyde was reported in the mid 1970s by Chadwick et al. ${ }^{34}$, Tam et al. ${ }^{35}$ and Kimura et $a l .{ }^{36}$ with the outermost valence cationic state located at $10.231 \mathrm{eV}$ ionization energy $^{37}$. Experimental studies of the ion fragmentation of acetaldehyde in coincidence with photoelectrons were presented in 1977 by Golovin et al. ${ }^{38}$ and in 1982 by Johnson et al. ${ }^{39}$. Subsequently, Keane et al. ${ }^{37}$ published in 1991 a study on the shake-up spectrum of this molecule. The same year Correia et $a l .{ }^{40}$ carried out an experimental and theoretical study of the normal Auger decay spectra of acetaldehyde with precise measurements of the core ionization energies. According to their work, the $\mathrm{O} 1 s$ ionization energy is $538.64 \mathrm{eV}$, and the formylic C1s and methylic C1s ionization energies are 294.45 and $291.80 \mathrm{eV}$, respectively. A more thorough assignment of the Auger spectra was presented in 1995 by Minelli et al. ${ }^{41}$.

In this work we report on a new experimental study of single-photon multiple ionization of acetaldehyde using a magnetic bottle multi-electron correlation spectrometer and compare our results, where possible, with existing theoretical predictions as well as previous experimental studies. In particular we present the valence double ionization electron spectrum of acetaldehyde in comparison with the known spectrum of normal Auger decay associated with the $\mathrm{O} 1 s$ core hole. This comparison gives us the possibility to assign the features observed in the valence double ionization electron spectrum.

In order to investigate site-specific effects we examine the single and double Auger spectra which are extracted by selecting events corresponding to a specific core-electron energy. In the case of single Auger decay we scrutinize the early theoretical predictions of Minelli et $a l .{ }^{41}$ which so far lacked comparison to suitably extracted experimental spectra. Selecting on the energy of the ejected electron(s) also allows us to investigate the fate of more exotic states such as shake-up and core-valence (sometimes called "shake-off") states. In particular, we compare the Auger decay of the shake-up states with the Auger decay of the ground state of the core ionized species. Similarly, the triple valence photoionization route is compared with both the double Auger decay of the core-ionized molecule and the Auger decay of corevalence doubly ionized acetaldehyde, since all three processes can lead to the same triply ionized final states. 


\section{EXPERIMENTAL AND ANALYSIS DETAILS}

The experiments were performed utilizing a versatile multi-electron coincidence technique based on a time-of-flight magnetic bottle spectrometer ${ }^{10,20,22,29}$. A key asset of this technique is the capability to reveal the energy correlation of multiple electrons originating from the same photoionization event. Electrons created in the light-matter interaction region of the spectrometer are guided by a combination of a strong and weak magnetic field ${ }^{42}$ through a flight tube of about $2 \mathrm{~m}$ length towards a multi-channel plate (MCP) detector with multi-hit capability.

The majority of the experiments were carried out at beam line U49/2 PGM-1 of the synchrotron radiation facility BESSY-II at the Helmholtz Zentrum, Berlin. The storage ring was operated in single bunch mode providing $30 \mathrm{ps}$ long light pulses at a repetition rate of about $1.25 \mathrm{MHz}$. In order to reduce the X-ray pulse repetition rate further to a level adequate for unambiguous time referencing of our set-up, a mechanical chopper ${ }^{43}$ was used, synchronized to the radio frequency signal of the storage ring. The experimental runs were carried out at the photon energies of $95 \mathrm{eV}, 350 \mathrm{eV}$ and $600 \mathrm{eV}$. Depending on the photon flux, the energy resolution of the monochromator was chosen to be about $300 \mathrm{meV}$ or better. In addition, we carried out auxiliary measurements in our home laboratory using a pulsed helium discharge lamp at the photon energies of $21.2 \mathrm{eV}(\mathrm{He}-\mathrm{I} \alpha)$ and $40.8 \mathrm{eV}(\mathrm{He}-\mathrm{II} \alpha)$.

The vapor pressure of acetaldehyde at room temperature is sufficiently high to create a suitable sample gas pressure in the spectrometer without the need for additional heating. We used a commercially available sample with a stated purity $>99 \%$ and used several freeze-pump-thaw cycles in order to remove air and other impurities. In order to verify the purity, we recorded conventional photoelectron spectra both in the valence and core regions and compared them to the reference spectra known from the literature ${ }^{34-37,39}$.

For the synchrotron radiation experiments, the Argon $\mathrm{L}_{2}$ and $\mathrm{L}_{3}$ photoelectron lines ${ }^{44}$ as well as the core ionization energies of acetaldehyde known from the literature ${ }^{40}$ were used to calibrate the flight time to kinetic energy conversion. For the helium lamp experiments the valence photoelectron spectrum of oxygen recorded at $21.2 \mathrm{eV}^{45}$ and the valence photoelectron spectrum of acetaldehyde itself $^{36}$ were used for calibration purposes. The photon energy of $95 \mathrm{eV}$ is sufficient for double photoionization involving all valence orbitals, but not core orbitals, while 350 and $600 \mathrm{eV}$ photons induce processes involving the core orbitals of 
the carbon and oxygen atoms. Thus, our analysis of processes involving the formation of carbon holes (both formylic and methylic) is based on the $350 \mathrm{eV}$ run, while for processes involving the oxygen electron, we used the data taken at the photon energy of $600 \mathrm{eV}$.

The time-of-flight to kinetic energy conversion is based on

$$
E_{k i n}=\frac{D^{2}}{\left(t-t_{0}\right)^{2}}+E_{0}
$$

where $t$ denotes the measured flight time and $E_{k i n}$ the electron kinetic energy, and where $D$ (which contains the length of the flight path), $t_{0}$ and $E_{0}$ are calibration parameters derived by least-square fitting of the features measured in the calibration spectra in comparison to the reference spectra.

The time resolution or bin size of the analog-to-digital converter was set to 100 ps. Because of the non-linearity of the conversion formula, the spectra and coincidence maps directly transformed to the energy scale would have non-constant bin size. In order avoid distortion of the intensity distributions, we have chosen a constant bin size of 0.2-1.0 eV (depending on the statistics and resolution in the particular cases) and redistributed the intensities on the basis of the degree of overlap between constant and non-constant bins. Thus, one electron can contribute to several neighboring bins conserving the sum of the proportions. The resulting coincidence map have electron kinetic energies on its axes. In order to obtain the double and triple ionization energy scales used in Figs. 1 and 8, and in Figs. 6 and 10, respectively, we changed the representation of the map to have the summed electron energies on one of the axes and then project the data onto this axis. The resulting spectrum then can be easily converted to the double or triple ionization energy scale by taking into account the photon energy used. The double ionization energy scale of the Auger electron spectra shown in Figs. 4 and 5 below, has been established by subtracting the kinetic energy of the Auger electron from the associated 1s ionization energy.

In the case of the double Auger process (see Fig. 6 below), we have selected triple coincidence events, where one of the electrons has the kinetic energy of the core electron of interest and where the kinetic energies of two other electrons fall outside this energy range. Then similarly to the single Auger case, the triple ionization energy scale of Auger pairs has been derived as the kinetic energy sum of electron pairs relative to the lowest single core ionization energy, where the sum has been deduced by shifting and projecting bins in the coincidence map akin to what has been described above for the cases of double valence and 
core-valence photoionization.

For the processes involving simultaneous emission of two electrons, we extracted distributions of the excess energy sharing which are comparable to differential cross sections as presented in the literature ${ }^{48-53}$. In the present work, a relative energy scale has been chosen. This means, we plot against the energy of each electron relative to the kinetic energy sum of both, because this quantity is normalized within each final state. This allows us to compare more easily the distributions corresponding to different peaks in the electron spectra. Also, we note that $0.3-1 \%$ on both edges of the diagrams were cut in order to exclude a sudden drop of the intensity to zero around the point where one of the electrons has zero kinetic energy.

\section{RESULTS AND DISCUSSION}

\section{A. Direct double valence photoionization}

In Fig. 1 valence double ionization electron spectra of acetaldehyde are shown as extracted from the coincidence data measured at the photon energies of $40.8 \mathrm{eV}$ and $95 \mathrm{eV}$, respectively. For direct comparison, the normal oxygen KLL Auger spectrum of acetaldehyde known from the literature ${ }^{40}$ is also included. It can be clearly seen that the spectra strongly resemble each other. The onset of double photoionization occurs at around $29 \mathrm{eV}$ in all three spectra. Essentially all of the spectral features known from the normal Auger spectrum are also observed in the valence double ionization electron spectra, but with different relative intensities due to the different nature of the ionization processes involved in forming the dicationic final states. The $95 \mathrm{eV}$ spectrum also contains some comparatively weak but sharp features in the higher ionization energy region which are not present in the oxygen Auger spectrum. They are putatively attributed to $\mathrm{N}_{4,5} \mathrm{OO}$ Auger decay of residual xenon atoms present from previous optimization work on the spectrometer. The two most apparent structures of this kind are labeled in Fig. 1 accordingly, and serve as an independent calibration reference. From the coincidence data at hand, we could extract the fraction of valence double photoionization to valence single photoionization as about $17 \%$ for the $40.8 \mathrm{eV}$ run.

The similarity between the normal KLL Auger spectrum of acetaldehyde recorded above 


\begin{tabular}{|c|c|c|c|}
\hline Peak & $\mathrm{DIE}(\mathrm{eV})$ & States & Leading configurations \\
\hline \multirow[t]{3}{*}{1} & 31.0 & ${ }^{1} A^{\prime}$ & $\left(10 a^{\prime}\right)^{-2}$ \\
\hline & & ${ }^{1,3} A^{\prime}$ & $\left(9 a^{\prime}\right)^{-1}\left(10 a^{\prime}\right)^{-1}$ \\
\hline & & ${ }^{1,3} A^{\prime \prime}$ & $\left(1 a^{\prime \prime}\right)^{-1}\left(10 a^{\prime}\right)^{-1}$ and $\left(2 a^{\prime \prime}\right)^{-1}\left(10 a^{\prime}\right)^{-1}$ \\
\hline 2 & 34.0 & ${ }^{1,3} A^{\prime \prime}$ & $\left(1 a^{\prime \prime}\right)^{-1}\left(10 a^{\prime}\right)^{-1}$ and $\left(2 a^{\prime \prime}\right)^{-1}\left(10 a^{\prime}\right)^{-1}$ \\
\hline 3 & 35.3 & ${ }^{1,3} A^{\prime}$ & $\left(8 a^{\prime}\right)^{-1}\left(10 a^{\prime}\right)^{-1}$ \\
\hline 4 & 37.3 & $1,3 A^{\prime}$ & $\left(7 a^{\prime}\right)^{-1}\left(10 a^{\prime}\right)^{-1}$ and $\left(6 a^{\prime}\right)^{-1}\left(10 a^{\prime}\right)^{-1}$ \\
\hline \multirow[t]{3}{*}{5} & 40.4 & ${ }^{1,3} A^{\prime \prime}$ & $\left(8 a^{\prime}\right)^{-1}\left(2 a^{\prime \prime}\right)^{-1}$ \\
\hline & & ${ }^{1} A^{\prime}$ & $\left(1 a^{\prime \prime}\right)^{-1}\left(2 a^{\prime \prime}\right)^{-1}$ and $\left(2 a^{\prime \prime}\right)^{-2}$ \\
\hline & & ${ }^{1} A^{\prime}$ & $\left(8 a^{\prime}\right)^{-2}$ and $\left(7 a^{\prime}\right)^{-1}\left(8 a^{\prime}\right)^{-1}$ \\
\hline \multirow[t]{3}{*}{ ‘6’ } & 41.6 & ${ }^{1,3} A^{\prime \prime}$ & $\left(7 a^{\prime}\right)^{-1}\left(2 a^{\prime \prime}\right)^{-1}$ and $\left(1 a^{\prime \prime}\right)^{-1}\left(9 a^{\prime}\right)^{-1}$ \\
\hline & & ${ }^{1,3} A^{\prime}$ & $\left(7 a^{\prime}\right)^{-1}\left(9 a^{\prime}\right)^{-1}$ \\
\hline & & $1,3 A^{\prime}$ & $\left(8 a^{\prime}\right)^{-1}\left(9 a^{\prime}\right)^{-1}$ \\
\hline \multirow[t]{4}{*}{7} & 43.3 & ${ }^{1,3} A^{\prime}$ & $\left(5 a^{\prime}\right)^{-1}\left(10 a^{\prime}\right)^{-1}$ \\
\hline & & ${ }^{1} A^{\prime}$ & $\left(7 a^{\prime}\right)^{-2}$ \\
\hline & & ${ }^{1,3} A^{\prime \prime}$ & $\left(6 a^{\prime}\right)^{-1}\left(2 a^{\prime \prime}\right)^{-1}$ \\
\hline & & ${ }^{1,3} A^{\prime \prime}$ & $\left(6 a^{\prime}\right)^{-1}\left(1 a^{\prime \prime}\right)^{-1}$ \\
\hline 8 & 46 & ${ }^{1,3} A^{\prime}$ & $\left(6 a^{\prime}\right)^{-1}\left(8 a^{\prime}\right)^{-1}$ \\
\hline 9 & 49 & ${ }^{1,3} A^{\prime}$ & $\left(5 a^{\prime}\right)^{-1}\left(8 a^{\prime}\right)^{-1}$ and $\left(6 a^{\prime}\right)^{-1}\left(7 a^{\prime}\right)^{-1}$ \\
\hline
\end{tabular}

TABLE I. States and leading configurations which contribute to the different features of the double valence ionization photoelectron spectrum of acetaldehyde shown in Fig. 1, making use of the works of Correia et al. ${ }^{40}$ and of Minelli et $a l .{ }^{41}$. 


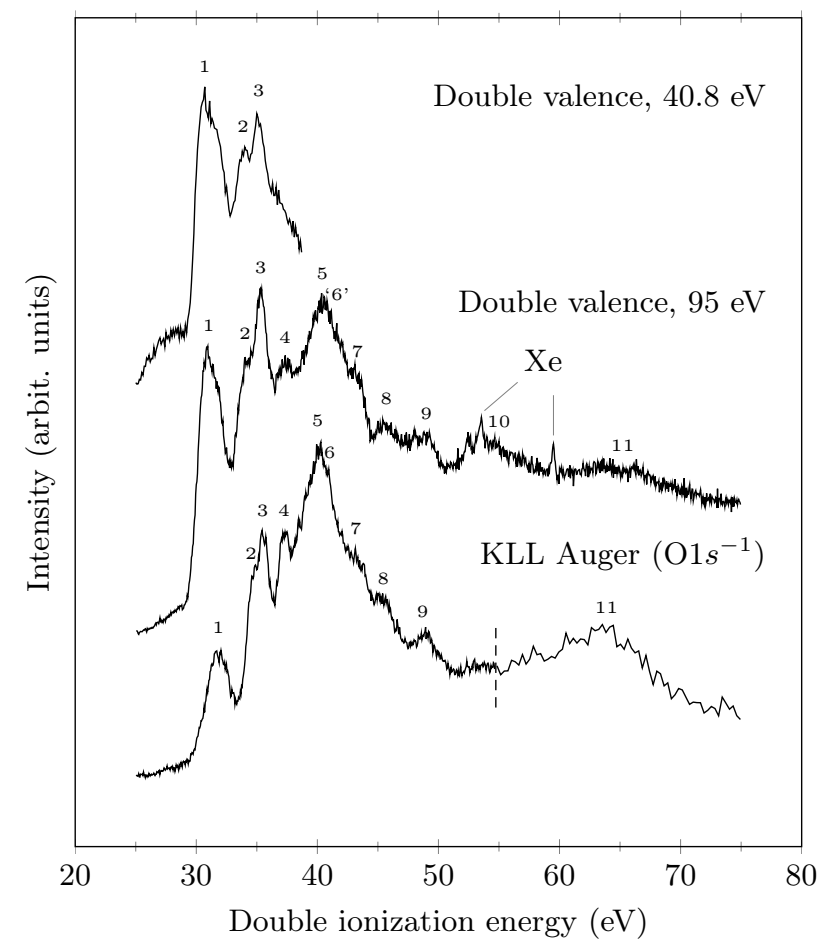

FIG. 1. Double valence ionization electron spectra of acetaldehyde recorded at the photon energies of 40.8 and $95 \mathrm{eV}$, in comparison to the oxygen KLL Auger spectrum of acetaldehyde known from the literature $^{40}$, which comprises two measurements of different resolution as indicated by the dashed line. Peak structures attributed to putative Auger processes in residual xenon atoms are labeled in the $95 \mathrm{eV}$ spectrum.

the O1s ionization edge and the valence double ionization electron spectra allows us to assign many of the features observed in the spectra reported here, in terms of the most significant electron configurations, by making use of the works of Correia et al. ${ }^{40}$ and of Minelli et al. ${ }^{41}$. The identifications are summarized in Table 1. It should be remembered that whereas the Auger decay process populates singlet spin states preferentially, the direct double ionization at $40.8 \mathrm{eV}$ and $95 \mathrm{eV}$, below the inner shell thresholds, is expected to populate triplets as well as singlets. As suggested by the calculations of Refs. ${ }^{40}$ and $^{41}$, the singlet-triplet splitting in this molecule is generally small in comparison to the instrumental resolution, so we believe that the assignments in terms of electron configurations given is adequate. Where the configurations include electrons taken from two different orbitals the probable presence of a triplet state in addition to the singlet is to be understood. In particular, peak 1 located at approximately $31.0 \mathrm{eV}$ ionization energy is associated with the lowest doubly ionized state 
${ }^{1} A^{\prime}$ of the $\left(10 a^{\prime}\right)^{-2}$ configuration which is a pure singlet, together with additional components formed from linear combinations of the configurations $\left(9 a^{\prime}\right)^{-1}\left(10 a^{\prime}\right)^{-1},\left(1 a^{\prime \prime}\right)^{-1}\left(10 a^{\prime}\right)^{-1}$ and $\left(2 a^{\prime \prime}\right)^{-1}\left(10 a^{\prime}\right)^{-1}$ which contribute to several final states, including triplets and singlets. As can be seen from Fig. 1, this peak is dominant in the $40.8 \mathrm{eV}$ spectrum, while in the $95 \mathrm{eV}$ spectrum it is less intense compared to its neighboring features. This change in relative intensity simply represents the normal increase in double photoionization cross sections with photon energy above threshold for each state. Feature 2, which appears as a partially resolved peak at approximately $34.0 \mathrm{eV}$ ionization energy in the $40.8 \mathrm{eV}$ spectrum and which is discernible as a shoulder in the $95 \mathrm{eV}$ spectrum, is formed primarily by the ${ }^{1,3} A^{\prime \prime}$ states of the $\left(1 a^{\prime \prime}\right)^{-1}\left(10 a^{\prime}\right)^{-1}$ and $\left(2 a^{\prime \prime}\right)^{-1}\left(10 a^{\prime}\right)^{-1}$ configurations. Peak 3 located at about $35.3 \mathrm{eV}$ ionization energy is well distinguishable in all three spectra. It is primarily associated with the ${ }^{1,3} A^{\prime}$ states of the $\left(8 a^{\prime}\right)^{-1}\left(10 a^{\prime}\right)^{-1}$ configuration and possibly the ${ }^{1,3} A^{\prime}$ states belonging to the $\left(1 a^{\prime \prime}\right)^{-1}\left(2 a^{\prime \prime}\right)^{-1}$. The main component of feature 4 which is observed at the ionization energy of around $37.3 \mathrm{eV}$ in the $95 \mathrm{eV}$ spectrum is likely to be formed by the ${ }^{1,3} A^{\prime}$ states connected to the $\left(7 a^{\prime}\right)^{-1}\left(10 a^{\prime}\right)^{-1}$ and $\left(6 a^{\prime}\right)^{-1}\left(10 a^{\prime}\right)^{-1}$ configurations.

Due to the increasing density of dicationic states at higher ionization energies, the structures labelled 5 and 7 are suggested to be formed by a number of almost equally strong electronic states. The most significant components for peak 5 located at the ionization energy of approximately $40.4 \mathrm{eV}$ can be associated to the ${ }^{1,3} A^{\prime \prime}$ state formed out of the $\left(8 a^{\prime}\right)^{-1}\left(2 a^{\prime \prime}\right)^{-1}$ configuration, a ${ }^{1} A^{\prime}$ state belonging to the $\left(1 a^{\prime \prime}\right)^{-1}\left(2 a^{\prime \prime}\right)^{-1}$ and $\left(2 a^{\prime \prime}\right)^{-2}$ configurations, and a ${ }^{1} A^{\prime}$ state characterized by the $\left(8 a^{\prime}\right)^{-2}$ and $\left(7 a^{\prime}\right)^{-1}\left(8 a^{\prime}\right)^{-1}$ configurations. The shoulder 6 barely discernible in case of the KLL Auger is even less noticeable on the double valence spectrum. The states contributing with intensities to peak 7 observed at the ionization energy of approximately $43.3 \mathrm{eV}$ are likely to be associated with the $\left(5 a^{\prime}\right)^{-1}\left(10 a^{\prime}\right)^{-1},\left(7 a^{\prime}\right)^{-2}$, $\left(6 a^{\prime}\right)^{-1}\left(2 a^{\prime \prime}\right)^{-1}$ and $\left(6 a^{\prime}\right)^{-1}\left(1 a^{\prime \prime}\right)^{-1}$ configurations.

In the case of features 8 and 9 at the ionization energies of around $46 \mathrm{eV}$ and $49 \mathrm{eV}$, respectively, a high density of states is expected to contribute where the dominant component is expected to belong to the $\left(6 a^{\prime}\right)^{-1}\left(8 a^{\prime}\right)^{-1}$ configuration for feature 8 and to the $\left(5 a^{\prime}\right)^{-1}\left(8 a^{\prime}\right)^{-1}$ and $\left(6 a^{\prime}\right)^{-1}\left(7 a^{\prime}\right)^{-1}$ configurations for feature 9 .

Two broad bands labeled 10 and 11 have so far not been interpreted. The latest seems to be present in both the $95 \mathrm{eV}$ spectrum and the oxygen KLL Auger spectrum as well as in the carbon KLL Auger spectrum (see feature 4 in Fig. 4 below and the Auger spectra 
presented by Correia et $\left.a l .{ }^{40}\right)$. We expect them to be populated by a fairly large number of dicationic states, possibly of repulsive nature.

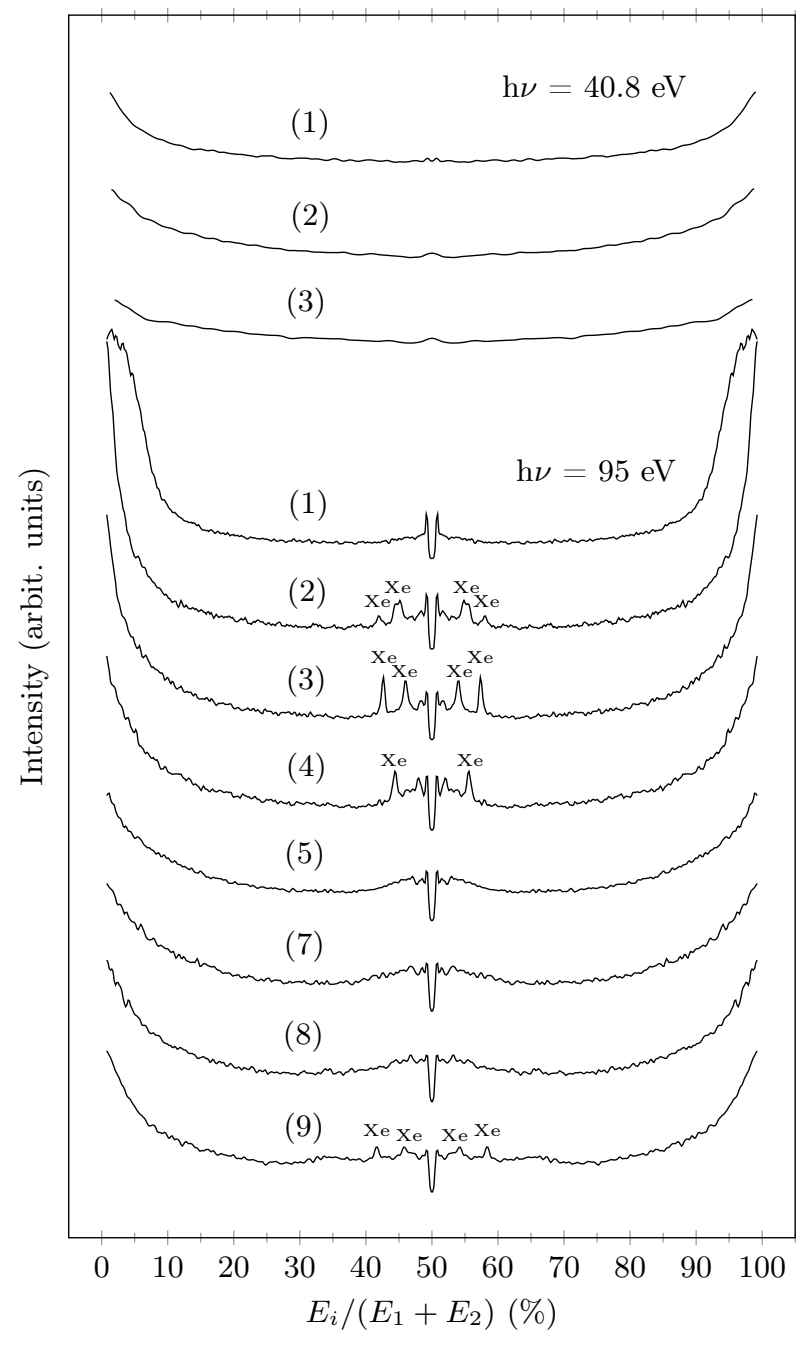

FIG. 2. Distributions of relative excess energy sharing for different final states reached by double valence ionization. The curves presented are based on the 40.8 and $95 \mathrm{eV}$ data and are normalized to the total intensity of the associated spectra. The curve labels correspond to the peak labels in Fig. 1. Sharp peak structures in the $95 \mathrm{eV}$ data are attributed to Auger decay in residual xenon atoms and are labeled accordingly.

In Fig. 2 we present distributions of the relative excess energy sharing for the different final states reached by double valence ionization; the curves displayed are based on the 40.8 and $95 \mathrm{eV}$ data. The curves are essentially structureless, apart from some sharp peaks close to the center of the distributions for $95 \mathrm{eV}$ case, which are attributed to $\mathrm{N}_{4,5} \mathrm{OO}$ Auger decay processes in xenon, and apart from the discontinuity at the center of the curves 
which is due to the dead time of the detector. This suggests that two valence electrons are emitted by acetaldehyde simultaneously rather than in a stepwise manner. Moreover, all the distributions reflect a U-shape as observed before for several other $\operatorname{cases}^{48-54}$. It is particularly well-pronounced for the curves with higher excess energy, while the curves with lower excess energies demonstrate an essentially flat form with only a small intensity increase at the edges. This pattern was predicted by several previous theoretical investigations ${ }^{51-53}$. The maximum excess energy, reached in the present data at $64.1 \mathrm{eV}$, is associated with the distribution 1 at $\mathrm{h} \nu=95 \mathrm{eV}$ and corresponds to the most pronounced U-shape. This case is attributed to the dominance of the "shake-off" mechanism ${ }^{55}$, where the second electron is released into the continuum by a "sudden" perturbation induced by the first ionization. The minimum excess energy of $5.6 \mathrm{eV}$ is found for the distribution 3 at $\mathrm{h} \nu=40.8 \mathrm{eV}$, and attributed to a decisive impact from the "knock-out" mechanism ${ }^{56}$, expressed as the process where the electron which initially absorbs the photon knocks out a second electron on its way to the continuum.

We also note, that the increase in intensity around the center of some of the $95 \mathrm{eV}$ distributions (in particular 4 - 7), i.e. equal energy sharing, are not attributed to the double valence ionization processes themselves, because in the coincidence maps these features are seen to gain intensity from interfering events of unknown origin at apparent energies below the lowest double ionization threshold.

\section{B. Normal carbon KLL Auger spectra of site-selectively ionized acetaldehyde}

The coincidence technique employed in the present work gives us the possibility to investigate the Auger decay associated with carbon atoms in chemically different surroundings (i.e. in the present case site-specifically for initial ionization either on the formylic and methylic $\mathrm{C} 1 s$ hole). This allows us to scrutinize theoretical predictions by Minelli et $a l_{.}{ }^{41}$ on site-specific Auger decay of acetaldehyde. The core photoelectron spectrum with the ionization energy scale relative to the methylic $\mathrm{C} 1 s$ threshold, obtained at the photon energy of $350 \mathrm{eV}$, is shown in the bottom panel of Fig. 3 (we will discuss in the next chapter the weaker shake-up peaks labeled with numbers). In order to obtain the Auger electron spectra of interest we selected double coincidence events where one of the electrons corresponds to one of two main peaks, i.e. it is either the formylic or the methylic C1s photoelectron, 


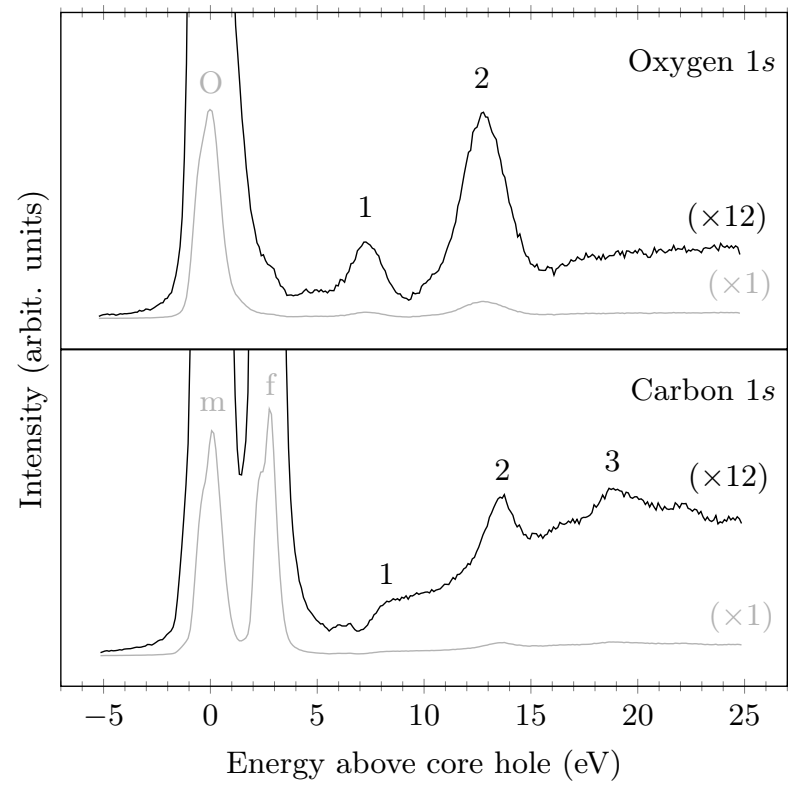

FIG. 3. Core photoelectron spectra of acetaldehyde with shake-up satellite structures for oxygen $1 s$ (upper panel) and carbon $1 s$ (lower panel) ionization. The horizontal scale shows the single ionization energy relative to the lowest core hole of each type. Both spectra are provided with two different intensity scales in order to bring out the satellite peaks as well as the main core ionization structures. Labels "O", "m" and "f" denote the main oxygen, methylic carbon and formylic carbon $1 s$ photoelectron lines, accordingly. The shake-up structures are labeled with numbers and their assignments are given in Table III, based on the work of Keane et al. ${ }^{37}$

and plotted the spectra of the second electron detected in pair. The results for the $350 \mathrm{eV}$ recording are shown in Figure 4 in direct comparison to the theoretical predictions of Minelli et $a l .{ }^{41}$.

As can be seen, the experimental Auger spectra extracted for the two different carbon atoms are quite different showing signatures of site-specificity. We note, that a similar difference between the experimental Auger spectra presented in Fig. 4 has been observed before in the Auger spectra associated with formylic and methylic C1s in acetone, as presented in the work by Eland et $a l .{ }^{32}$. In comparing our experimental result with the known theoretical spectra, we note a reasonable agreement, bearing in mind the limited resolving power of the magnetic bottle spectrometer at such comparatively high kinetic energies. A shift of essential parts of the theoretical spectra towards lower ionization energies of about $2 \mathrm{eV}$ is visible, which is not unexpected when comparing the theoretical work of Minelli et al. ${ }^{41}$ 


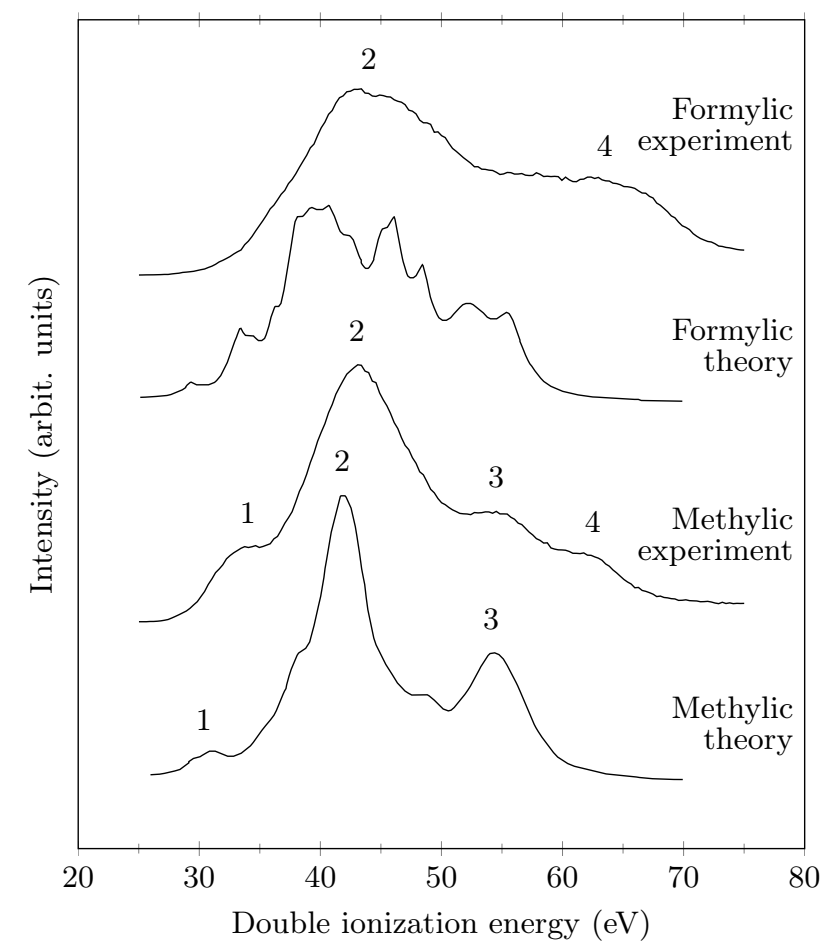

FIG. 4. KLL Auger electron spectra of acetaldehyde associated with the formation of the methylic and the formylic $\mathrm{C} 1 s$ hole $(\mathrm{h} \nu=350 \mathrm{eV})$. Corresponding theoretical spectra, taken from the work of Minelli et al. ${ }^{41}$, are included for comparison.

with the original experimental Auger data of Correia et $a{ }^{40}{ }^{40}$

The methylic spectrum shows several distinct features in relatively good agreement with the theoretical spectrum, which allows us to assign those features as summarized in Table 2. According to the work of Minelli et al. ${ }^{41}$ feature 1 arises mainly from transitions to the final states ${ }^{3} A^{\prime},{ }^{3} A^{\prime \prime},{ }^{1} A^{\prime}$ and ${ }^{1} A^{\prime \prime}$, most likely primarily related to vacancies in the outermost valence orbitals. Since the density of states increases at higher double ionization energies, both features 2 and 3 are expected to consist of many overlapping states. The most significant final states for feature 2 are three ${ }^{1} A^{\prime}$ states associated with the $\left(7 a^{\prime}\right)^{-1}\left(8 a^{\prime}\right)^{-1},\left(1 a^{\prime \prime}\right)^{-2}$ and $\left(6 a^{\prime}\right)^{-1}\left(9 a^{\prime}\right)^{-1}$ configurations, and one ${ }^{1} A^{\prime \prime}$ state associated with the leading configurations $\left(7 a^{\prime}\right)^{-1}\left(2 a^{\prime \prime}\right)^{-1}$ and $\left(9 a^{\prime}\right)^{-1}\left(1 a^{\prime \prime}\right)^{-1}$. In case of feature 3 two ${ }^{1} A^{\prime}$ states and four ${ }^{1} A^{\prime \prime}$ states, which involve the leading configurations $\left(5 a^{\prime}\right)^{-1}\left(9 a^{\prime}\right)^{-1},\left(6 a^{\prime}\right)^{-1}\left(7 a^{\prime}\right)^{-1},\left(5 a^{\prime}\right)^{-1}\left(1 a^{\prime \prime}\right)^{-1}$ and $\left(5 a^{\prime}\right)^{-1}\left(2 a^{\prime \prime}\right)^{-1}$, are most significant. So far, there is no interpretation for feature 4 .

Akin to what was done above, from the coincidence data at hand, we extracted the total intensity of the methylic carbon Auger spectrum relative to the total intensity of the 


\begin{tabular}{|c|c|c|c|}
\hline Peak & DIE $(\mathrm{eV})$ & States & Leading configurations \\
\hline \multirow[t]{4}{*}{1} & 34 & ${ }^{3} A^{\prime}$ & \\
\hline & & ${ }^{3} A^{\prime \prime}$ & \\
\hline & & ${ }^{1} A^{\prime}$ & \\
\hline & & ${ }^{1} A^{\prime \prime}$ & \\
\hline \multirow[t]{4}{*}{2} & 43 & ${ }^{1} A^{\prime}$ & $\left(7 a^{\prime}\right)^{-1}\left(8 a^{\prime}\right)^{-1}$ \\
\hline & & ${ }^{1} A^{\prime}$ & $\left(1 a^{\prime \prime}\right)^{-2}$ \\
\hline & & ${ }^{1} A^{\prime}$ & $\left(6 a^{\prime}\right)^{-1}\left(9 a^{\prime}\right)^{-1}$ \\
\hline & & ${ }^{1} A^{\prime \prime}$ & $\left(7 a^{\prime}\right)^{-1}\left(2 a^{\prime \prime}\right)^{-1}$ and $\left(9 a^{\prime}\right)^{-1}\left(1 a^{\prime \prime}\right)^{-1}$ \\
\hline \multirow[t]{4}{*}{3} & 54 & ${ }^{1} A^{\prime}$ & $\left(5 a^{\prime}\right)^{-1}\left(9 a^{\prime}\right)^{-1}$ \\
\hline & & ${ }^{1} A^{\prime}$ & $\left(6 a^{\prime}\right)^{-1}\left(7 a^{\prime}\right)^{-1}$ \\
\hline & & ${ }^{1} A^{\prime \prime}$ & $\left(5 a^{\prime}\right)^{-1}\left(1 a^{\prime \prime}\right)^{-1}$ \\
\hline & & ${ }^{1} A^{\prime \prime}$ & $\left(5 a^{\prime}\right)^{-1}\left(2 a^{\prime \prime}\right)^{-1}$ \\
\hline
\end{tabular}

TABLE II. States and leading configurations which contribute, according to the work of Minelli et $a l^{41}$, to the different peaks of the normal Auger decay spectra of acetaldehyde shown in Fig. 4, formed upon formylic and methylic $1 s$ hole creation.

formylic Auger spectrum where a relative increase of about $4 \%$ is observed. This verifies the prediction of Minelli et al. ${ }^{41}$ that the Auger decay associated with the methylic carbon $1 s$ hole should be stronger than the Auger decay associated with the formylic $1 s$ hole.

\section{Normal Auger decay of shake-up states in core-ionized acetaldehyde}

In removal of a core electron, an additional electron may be excited into an unoccupied valence orbital, which leads to the formation of shake-up or satellite states located at higher ionization energies. In Fig. 3 we display the shake-up satellite spectra obtained in the vicinity of $\mathrm{O} 1 s$ and $\mathrm{C} 1 s$ core ionized acetaldehyde. Similar spectra were investigated before by Keane et al. ${ }^{37}$, but the Auger decay of those shake-up states, which we will present below, 
Peak

1

2

12.94

7.5
Leading configurations

$$
\left(1 a^{\prime}\right)^{-1}\left(2 a^{\prime \prime}\right)^{-1} 3 a^{\prime \prime}
$$

$\left(1 a^{\prime}\right)^{-1}\left(1 a^{\prime \prime}\right)^{-1} 3 a^{\prime \prime}$

$\left(1 a^{\prime}\right)^{-1}\left(10 a^{\prime}\right)^{-1} 11 a^{\prime}$

Shake-up states involving the carbon $1 s\left(2 a^{\prime}\right.$ or $\left.3 a^{\prime}\right)$ shells

Peak

1

2

3
Shake-up energy (eV)

7.5

18.97
Leading configurations

$$
\left(2 a^{\prime}\right)^{-1}\left(2 a^{\prime \prime}\right)^{-1} 3 a^{\prime \prime}
$$

$$
\begin{gathered}
\left(2 a^{\prime}\right)^{-1}\left(1 a^{\prime \prime}\right)^{-1} 3 a^{\prime \prime} \\
\left(2 a^{\prime}\right)^{-1}\left(10 a^{\prime}\right)^{-1} 13 a^{\prime}
\end{gathered}
$$

$$
\begin{aligned}
& \left(3 a^{\prime}\right)^{-1}\left(1 a^{\prime \prime}\right)^{-1} 4 a^{\prime \prime} \\
& \left(3 a^{\prime}\right)^{-1}\left(8 a^{\prime}\right)^{-1} 14 a^{\prime} \\
& \left(3 a^{\prime}\right)^{-1}\left(8 a^{\prime}\right)^{-1} 15 a^{\prime} \\
& \left(3 a^{\prime}\right)^{-1}\left(10 a^{\prime}\right)^{-1} 16 a^{\prime}
\end{aligned}
$$

TABLE III. Leading configurations contributing to the shake-up satellite features observed in Fig. 3, according to the work of Keane et $a l .{ }^{37}$ and partially making use of Refs. ${ }^{46,47}$. For the $\mathrm{C} 1 s$ shake-up features, the energies are given relative to the methylic threshold regardless of their interpretation.

remained so far unobserved.

Briefly, as can be seen in the lower panel of Fig. 3, the shake-up spectrum of carbon $1 s$ ionized acetaldehyde consists of 3 features. Two of them are located at ionization energies shifted by about $4.85 \mathrm{eV}$ and $10.85 \mathrm{eV}$ above the formylic $1 s$ ionization threshold and are 


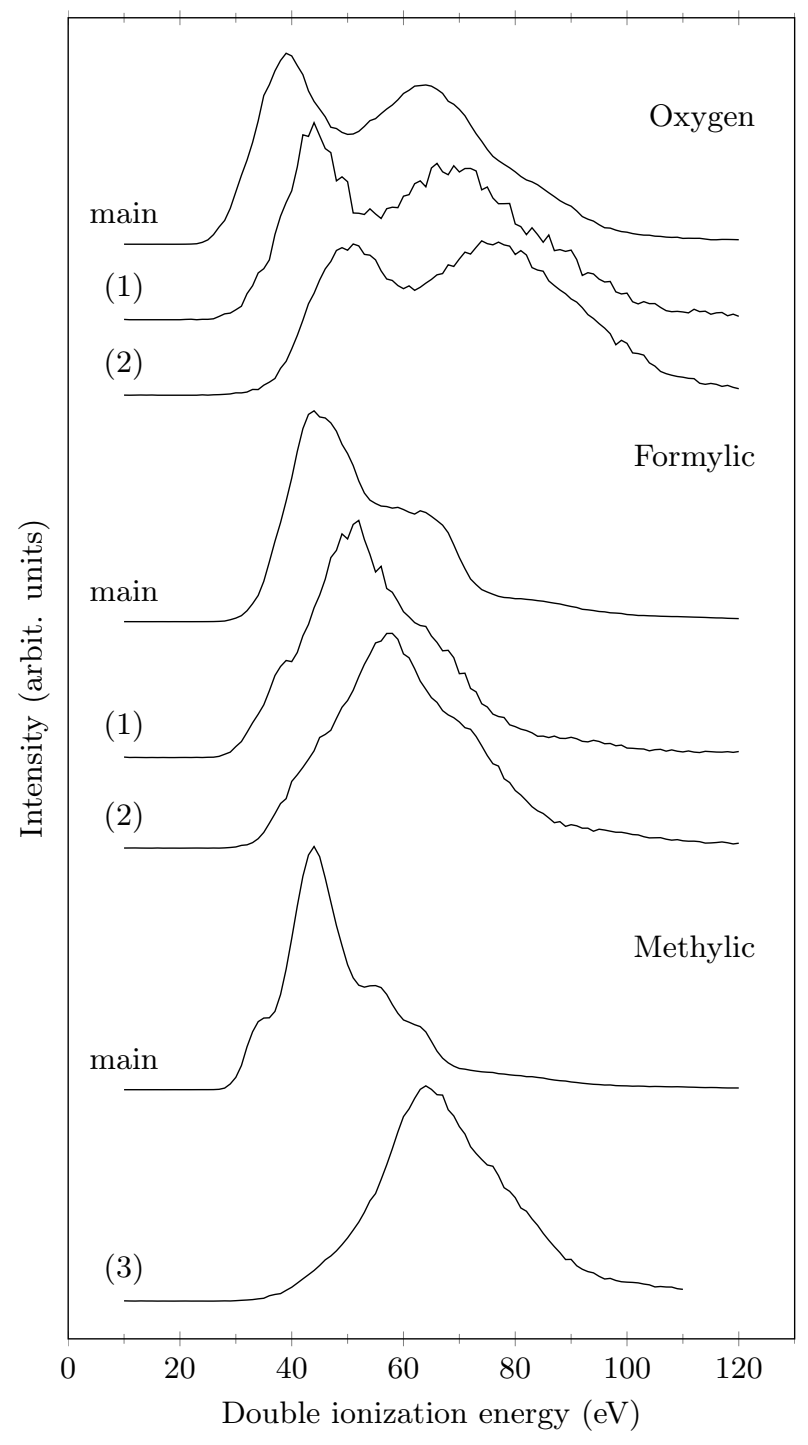

FIG. 5. KLL Auger electron spectra of core ionized shake-up states of acetaldehyde in comparison to the Auger spectra of the associated $\mathrm{O} 1 s$ (upper panel) and $\mathrm{C} 1 s$ (middle and lower panel) ionized ground states denoted as "main". The spectra labels correspond to the peak labels in Fig. 3.

associated in the work of Keane et al. ${ }^{37}$ with shake-up processes involving the formylic core hole, while the third structure, located at about $18.95 \mathrm{eV}$ above the methylic $1 s$ core ionization threshold, is suggested in Ref. 37 to be a satellite of the methylic core hole. The first peak of the low energy satellite structure is ascribed to a triplet parentage and is not assigned to any particular transition. The second structure corresponds mainly to a transition from the $1 a^{\prime \prime}$ orbital (being delocalized due to hyperconjugation, with about equal contributions from the formylic and methylic groups) to the delocalized anti-bonding $3 a^{\prime \prime}$ 
orbital. Also, according to the work of Keane et al. ${ }^{37}$ it has a significant contribution from a $10 a^{\prime} \rightarrow 13 a^{\prime}$ transition. The comparatively high ionization energy satellite associated with the methylic $1 s$ hole seems to involve transitions to higher valence or Rydberg orbitals.

The oxygen $1 s$ shake-up spectrum of acetaldehyde displayed in the upper panel of Fig. 3 exhibits two distinct states located at about $12.94 \mathrm{eV}$ and $7.5 \mathrm{eV}$ above the oxygen $1 s$ ionization threshold. They are described in the work of Keane et al. ${ }^{37}$ as mainly a transition from the $1 a^{\prime \prime}$ orbital, localized on formylic group to the anti-bonding $3 a^{\prime \prime}$ orbital with a small contribution from the $10 a^{\prime} \rightarrow 11 a^{\prime}$ transition and as the transition from the $2 a^{\prime \prime}$ orbital localized on the methylic group to the same antibonding $3 a^{\prime \prime}$ orbital, respectively.

In order to extract the associated Auger spectra, we selected double electron coincidence events with the kinetic energy of one of the electrons within the range of the shake-up satellite spectrum and plotted the intensity distribution of the second electron measured in pair. Such spectra are shown in Fig. 5 which also include the Auger decay spectra of the corresponding core-ionized ground states denoted as "main".

In comparing the three Auger spectra associated with the decay of the oxygen $1 s$ core hole as shown in the upper panel of Fig. 5, we see that all three spectra are very similar in shape, but that the dicationic final states reached by Auger decay of the first and second shake up states (features 1 and 2 in the upper panel of Fig. 5) are shifted towards higher double ionization energies. The shifts correspond to the associated shake-up energies of the peaks, except for the onsets, which are shifted by $4 \mathrm{eV}$ less than the main features. This suggests the dominance of spectator decay, not involving directly the initially excited electron in the Auger process. In contrast, the lower shift in the case of the onsets may reflect contributions from participator decay.

As in the oxygen case, the Auger spectra associated with the carbon $1 s$ shake-up states are also shifted towards higher ionization energies as can be seen in the middle and lower panel of Fig. 5. However, in contrast to the oxygen case, the spectral shapes differ quite substantially in the carbon Auger cases. Again, the shifts of the main features are essentially equal to the shake-up energies. The shifts of the onsets are 1.5, 4 and $9 \mathrm{eV}$ for spectra (1), (2) and (3), respectively, and are notably smaller than the corresponding shake-up energies. Similar as for the oxygen case, this suggests contributions from participator decay for the onsets and from spectator decay for the other features. 


\section{Double Auger decay of carbon and oxygen $1 s$ ionized acetaldehyde}

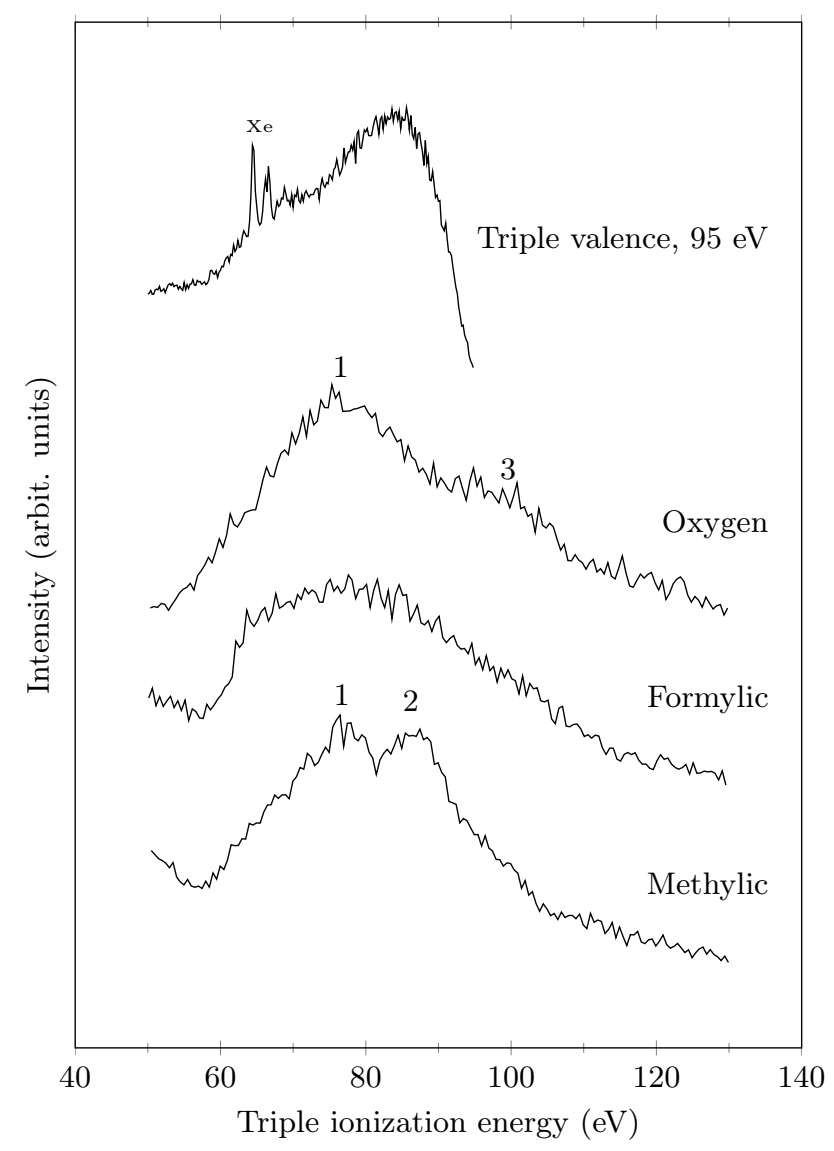

FIG. 6. KLLL double Auger electron spectra associated with the formation and decay of the oxygen $1 s$, formylic $1 s$ and methylic $1 s$ core holes in acetaldehyde. For comparison the triple valence electron spectrum obtained at the photon energy of $95 \mathrm{eV}$ is included. Sharp peak structures attributed to putative Auger processes in residual xenon atoms are labeled in the $95 \mathrm{eV}$ spectrum.

The decay of a core hole may lead to the ejection of not only one, but two or more Auger electrons. The case of two-electron emission is commonly denoted in the literature as (KLLL) double Auger decay leading to electronic states with three vacancies in the valence shells. Spectra of this kind associated with the formation and decay of methylic $1 s$, formylic $1 s$ and oxygen $1 s$ core holes in acetaldehyde are presented in Fig. 6. Since both KLLL double Auger decay and triple valence photoionization can populate the same tricationic final states, we included in the upper panel of Fig. 6 a triple valence electron spectrum recorded at the photon energy of $95 \mathrm{eV}$ for direct comparison. In this spectrum, there are two sharp features present which possibly originate from residual xenon atoms. The abrupt 


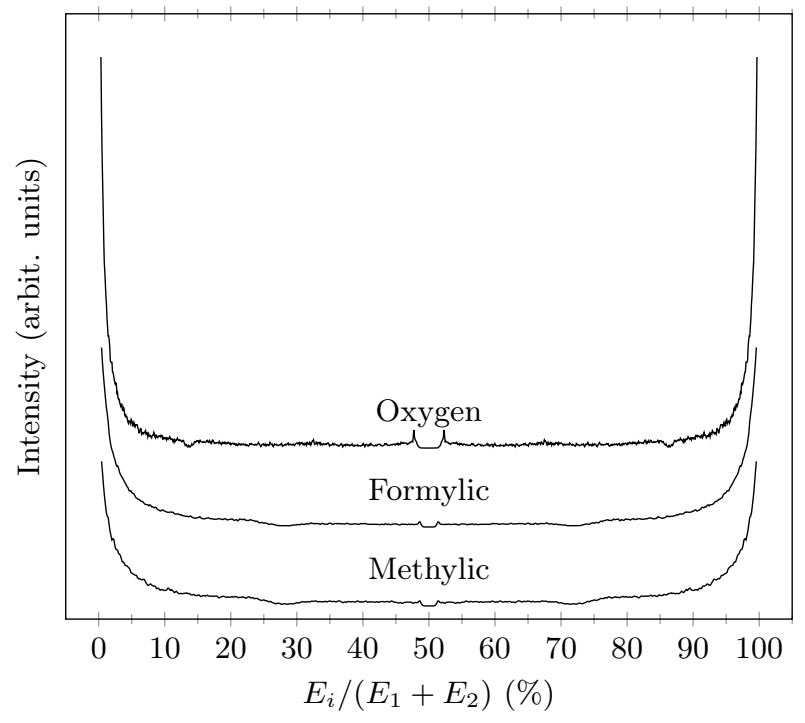

FIG. 7. Distributions of relative excess energy sharing for the double Auger decay of methylic $1 \mathrm{~s}$ (lower panel), formylic $1 s$ (middle panel) and oxygen $1 s$ (upper panel) core holes in acetaldehyde.

cut-off on the higher ionization energy side of this spectrum is due to the chosen photon energy.

As can be seen from these spectra, the onset of triple ionization of acetaldehyde appears in most of the cases at around $58 \mathrm{eV}$, save for the oxygen double Auger spectrum which seems to strech to even lower ionization energies. The spectra are essentially structureless, with one clear exception; in the methylic double Auger spectrum, we can discern two structures located at about $75 \mathrm{eV}$ and $85 \mathrm{eV}$ triple ionization energy labelled 1 and 2. Structure 1 seems also to be identifiable in the O1s double Auger spectrum shown in the middle panel of Fig. 6. In addition to that, a new, third structure located at about $95 \mathrm{eV}$ also seems to be present in the methylic spectrum as a shoulder on the high energy side of structure 2 .

A possible explanation for these structures could be the involvement of different valence vacancies forming those states. I.e. we expect band 1 to be formed primarily by final states with two vacancies in the oxygen lone-pair orbital, while bands 2 and 3 might have one or no vacancy in this orbital, respectively. The general lack of sharp peak structures in all three spectra might be related to the fact that the number of possible states formed out of triple valence vacancies are substantially larger than the number of doubly ionized states discussed before. Furthermore, it can be expected that the majority of the triply-ionized states are repulsive. 
In order to analyze the double Auger processes in acetaldehyde further, we derived the relative excess energy distributions of the two Auger electrons emitted upon the decay of the different core holes as shown in Fig. 7. Since the Auger spectra are essentially structureless, the distributions shown are integrated over the triple ionization energy range of 59-105 eV.

As can be seen, in all three cases, the distributions are essentially continuous which implies that the two Auger electrons share their energy arbitrarily and are therefore regarded as ejected primarily in a direct process. In comparing the distributions with each other, we noticed that double Auger processes associated with the two different $\mathrm{C} 1 s$ vacancies do not demonstrate any significant discrepancy in the energy sharing, which is probably due to the fact, that they are very close in energy. The oxygen distribution demonstrates steeper form, which implies higher excess energy of the oxygen double Auger decay compared to the same process involving the carbon atoms. This finding is similar to the results discussed above within section III A and to some of the cases known in the literature ${ }^{51-53}$.

In the distribution for the oxygen case, dips appear at around $13.5 \%$ and $86.5 \%$. Similarly, for the carbon cases dips appear at $28 \%$ and $72 \%$. Those features are due to excluded events where more than one electron has the kinetic energy which corresponds to the ejected $1 s$ photoelectron. This overlap leads to an ambiguity in distinguishing one of the Auger electrons and the photoelectron. Moreover, there are some weak bumps in the oxygen distribution at about $32.5 \%$ and $67.5 \%$ which we traced back to accidental coincidence events with the involvement of a carbon $1 s$ photoelectron.

In comparing the total fraction of double Auger decay associated with the methylic $1 s$ vacancy to the total fraction of double Auger decay associated with the formation of a formylic $1 s$ core hole, we find that the latter is about 1.2 times stronger. This stands in clear contrast to our finding for site-selective normal Auger decay of the same core vacancies (cf. section IIIB). The branching ratios of double Auger decay with respect to the single Auger process is estimated as about 29\%, 27\% and $21.5 \%$ for the oxygen, formylic and methylic carbon $1 s$ core hole, respectively.

\section{E. Normal Auger decay of core-valence states in core-ionized acetaldehyde}

Upon single photon absorption, a valence and a core electron may be ejected simultaneously, a process which is denoted as core-valence double ionization or "shake-off". This 


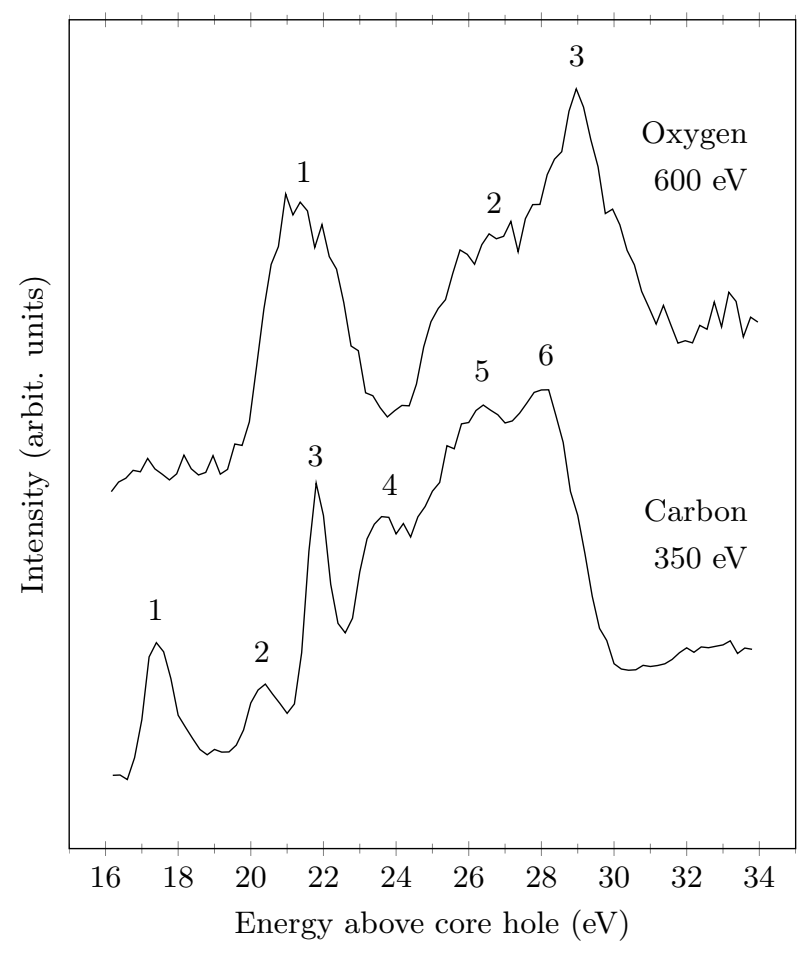

FIG. 8. Lower panel: core-valence double ionization electron spectrum of acetaldehyde measured at $h \nu=350 \mathrm{eV}$, i.e. in the vicinity of the formylic $(294.45 \mathrm{eV})$ and methylic $(291.8 \mathrm{eV}) 1 s$ core ionization thresholds. Upper panel: core-valence double ionization electron spectrum of acetaldehyde measured at $h \nu=600 \mathrm{eV}$, i.e. in the vicinity of the oxygen $1 s(538.64 \mathrm{eV})$ threshold. The energy scale shows the double ionization energy relative to the lowest core hole of each type.

process can also be followed by the emission of an Auger electron leading again to states with three valence vacancies. Similarly to the case of valence double ionization, the two electrons emitted in the primary core-valence ionization step can share the photon energy arbitrarily. This fact makes it impossible to distinguish the origin of the two electrons involved in the double ionization step, which implies especially in the case of acetaldehyde that it is impossible to obtain experimentally site-selective core-valence double ionization spectra for the two chemically different carbon atoms.

To extract the core-valence ionization spectrum of acetaldehyde involving the carbon edges, we used the data set obtained at $350 \mathrm{eV}$, and in order to extract the core-valence ionization spectrum which involves the oxygen core level we used the data set recorded at $600 \mathrm{eV}$. The spectra were derived by selecting from the triple coincidences those events which contain one electron in the range of the normal carbon and oxygen Auger spectra, respec- 


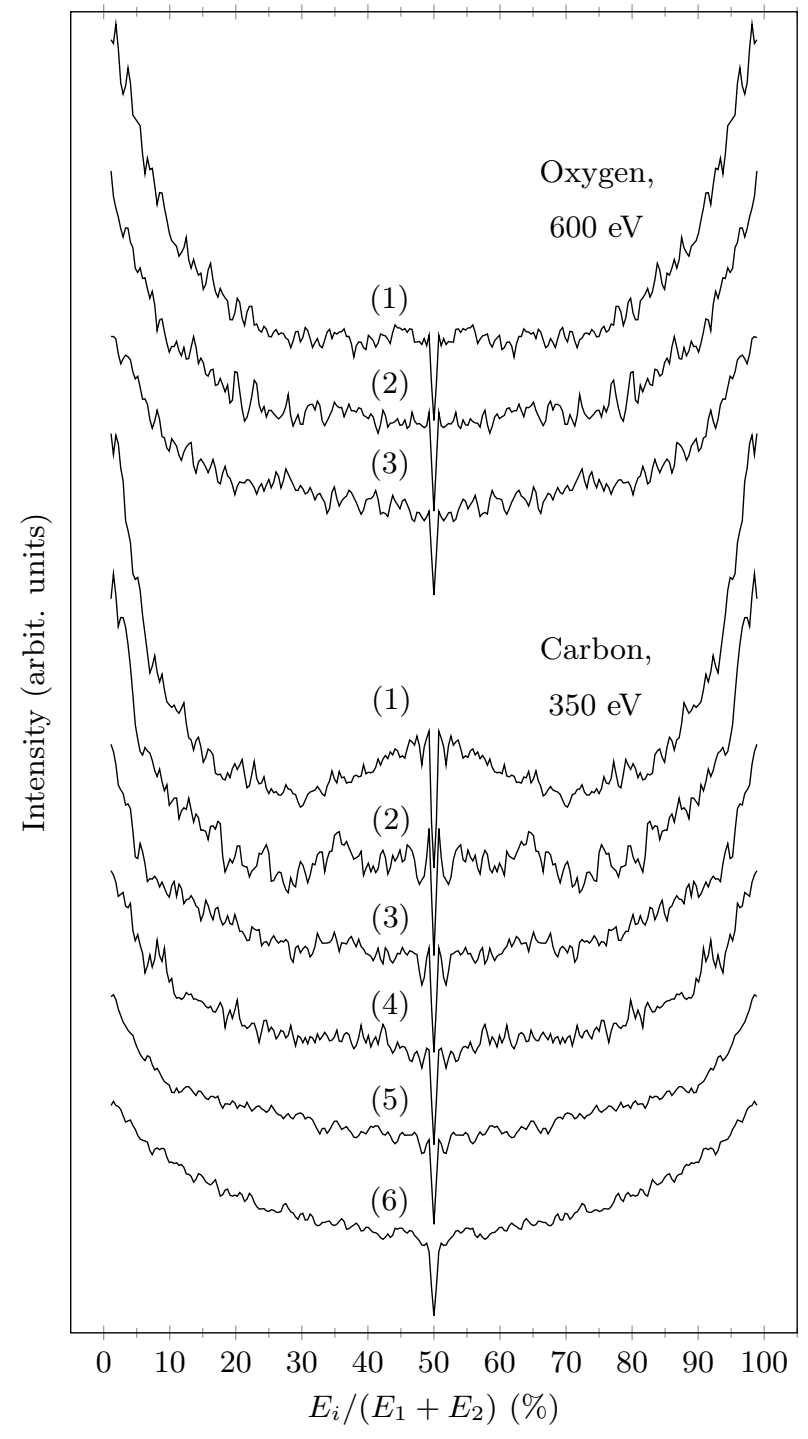

FIG. 9. Distributions of relative excess energy sharing for core-valence ionization involving carbon $(\mathrm{h} \nu=350 \mathrm{eV})$ and oxygen $(\mathrm{h} \nu=600 \mathrm{eV}) 1 s$ electrons. The distributions are numbered according to the structures observed in Fig. 8.

tively. The double photoionization spectra reflecting the associated electron pairs relative to the corresponding core level thresholds can then be plotted accordingly as displayed in Fig. 8. Very recently, those spectra were examined in detail in a separate work ${ }^{57}$ from where we adapted the assignments given in Table IV.

In order to examine further the core-valence states observed in Fig. 8, we present in Fig. 9 distributions of relative excess energy sharing both for the oxygen $1 s$ and the carbon $1 s$ core-valence states. As can be seen from this figure, similarly to Figs. 2 and 7 , we 
observe mainly again continuous U-shape distributions. In going from the higher to lower core-valence states we see that the distributions become more strongly pronounced at higher excess energies. This verifies the predictions by Hoszowska et al. ${ }^{58}$ that similar mechanisms should govern core-valence and double valence photoionization processes. We note that in the case of distribution 1 of the carbon case, the region of enhanced intensity around equal energy sharing is reminiscent of what has been found above in the case of the distributions associated with double valence ionization displayed in Fig. 2. It seems again to be associated with events well below the lowest core-valence double ionization energy, which origin are unclear.

Finally, in order to cast light onto the decay of the core-valence states observed in Fig. 8, Auger spectra associated with the formation of the different core-valence states are shown in Fig. 10. As can be seen from this figure, the spectra corresponding to the decay of the different $\mathrm{C} 1 s$-valence double hole states are shifted relative to each other. Similarly, the spectra corresponding to the decay of the different O1s-valence double hole states are shifted relative to each other. The shifts observed are equal to the energy difference between the corresponding core-valence states. In other words; if the spectra are plotted on the kinetic energy scale instead, the spectral features line up. As can be seen from Fig. 10, the Auger processes following the core-valence photoionization involving the carbon $1 s$ holes populate

predominantly the lower triply ionized states starting at about the same triple ionization energy as the valence triple ionization spectrum recorded at $95 \mathrm{eV}$ (included in the upper panel) and the double Auger spectra discussed above (58 eV, see section IIID). Since the maxima observed fall within the ionization energy range of band 1 in Fig. 6, this may suggest the primary involvement of two electrons from the oxygen lone-pair $\left(10 a^{\prime}\right)$ orbital to the final state formation. In contrast, the Auger spectra of the oxygen core-valence states appear to have their maxima at higher triple ionization energies, roughly in the same region as band 3 in Fig. 6 where the involvement of the oxygen lone-pair $\left(10 a^{\prime}\right)$ orbital is less likely.

\section{CONCLUSIONS}

Single-photon multiple ionization processes of acetaldehyde involving valence and core levels have been investigated by means of a multi-particle coincidence technique based on the time-of-flight magnetic bottle principle. The double valence ionization electron spectrum 


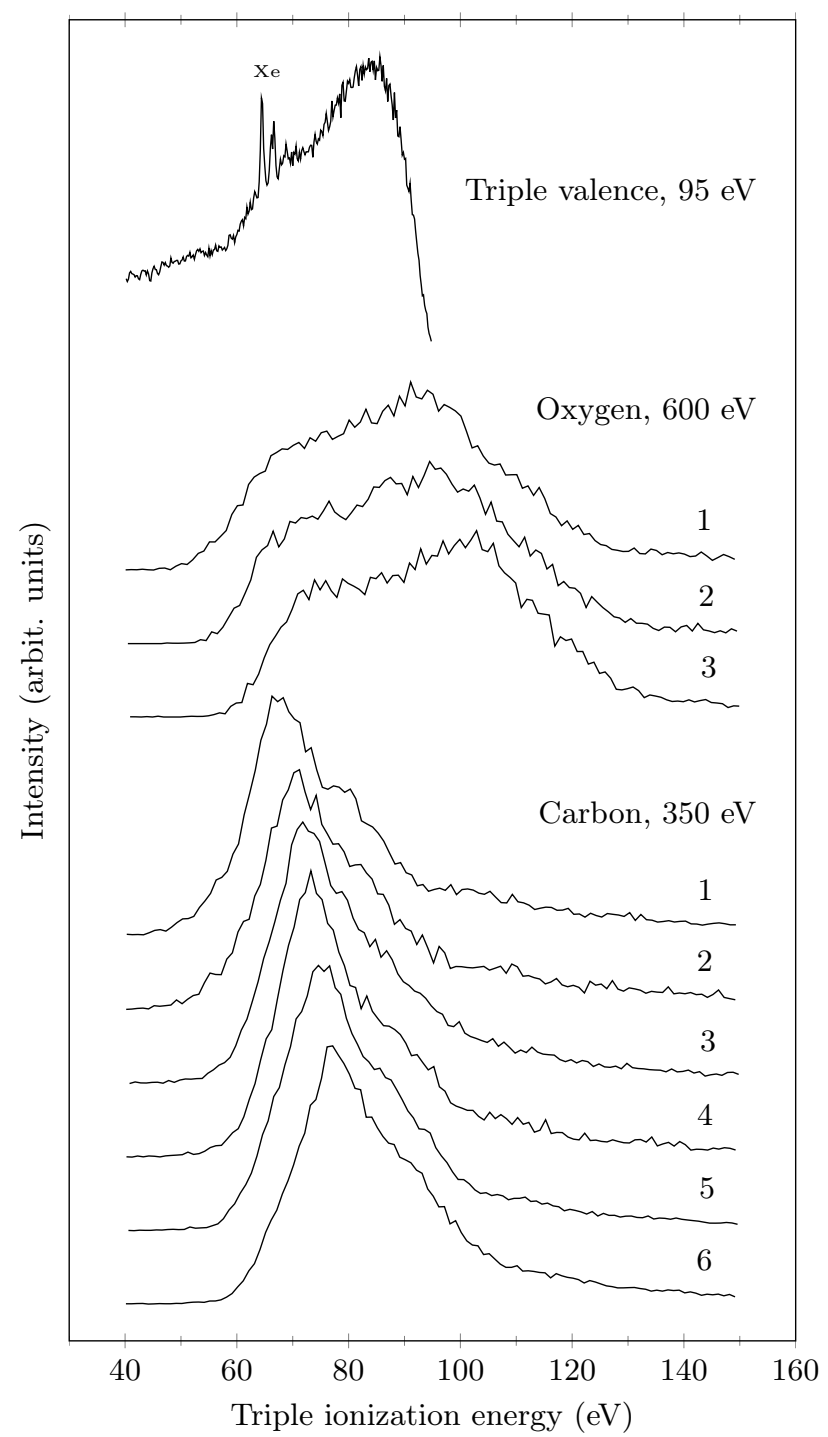

FIG. 10. KLL Auger electron spectra of core-valence states of acetaldehyde. The spectra are numbered according to the peaks in Fig. 8. The triple valence photoionization spectrum is given for comparison.

of acetaldehyde was found to be very similar to the known oxygen KLL Auger spectrum of acetaldehyde $^{40}$ which allowed us to assign the features observed using the calculations by Minelli it et al. ${ }^{41}$. The similarity suggests the involvement of the same valence orbitals.

Single Auger decay spectra were extracted site-selectively for the case of the carbon core holes. The spectra associated with the formation of the formylic and methylic $1 s$ core holes were found to be substantially different from each other, and are in good agreement with the theoretical predictions of by Minelli it et al. ${ }^{41}$. A similar distinction was found for the 
double Auger spectra of acetaldehyde for which no theoretical predictions exist so far. The observed differences suggest that the localization of the core hole significantly affects the involvement of the different orbitals in the Auger process.

Spectra reflecting tricationic states of acetaldehyde formed by double Auger decay of the $1 s$ core holes, by single Auger decay of core-valence doubly ionized states or by the direct removal of three valence electrons, were obtained for the first time and were found to be essentially structureless. Furthermore, Auger decay spectra of shake-up satellite states in acetaldehyde were presented for the first time and showed that the spectator process being dominant.

Moreover, in examining the energy sharing of two-electron emission processes of acetaldehyde involving either solely valence or valence and core orbitals, U-shape distributions were revealed in good agreement with previous findings in the literature for similar cases. ${ }^{4-54}$. Akin to those previous studies for other systems, the energy is shared more equally for cases when the excess energy is low (i.e. ionization energy is close to the photon energy chosen) which implies the knock-out mechanism being more dominant than the shake-off mechanism.

\section{ACKNOWLEDGMENTS}

This work has been financially supported by the Swedish Research Council (VR) and the Knut and Alice Wallenberg Foundation, Sweden. We thank Svante Svensson for providing the original data of the work by Correia et $a l .{ }^{40}$, which were used in parts of Fig. 1 . We would like to warmly acknowledge the support by the staff and colleagues at the Helmholtz Centre for Materials and Energy GmbH BESSY-II, Berlin. This work was also supported by the European Community - Research Infrastructure Action under the FP6 "Structuring the European Research Area" Programme (through the Integrated Infrastructure Initiative "Integrating Activity on Synchrotron and Free Electron Laser Science" - Contract R II 3-CT-2004-506008).

\section{REFERENCES}

${ }^{1}$ K. Siegbahn et al., North-Holland Pub. Co., ESCA applied to free molecules (1970). 
${ }^{2}$ R. Spohr, T. Bergmark, N. Magnusson, L.O. Werme, C. Nordling and K. Siegbahn, Physica Scripta 1-2, 31 (1970).

${ }^{3}$ L. Karlsson, L.O. Werme, T. Bergmark and K. Siegbahn, J. Electr. Spectr. Rel. Phen. 3, 181 (1974)

${ }^{4}$ R.W. Shaw, J.S. Jen and T.D. Thomas, J. Electr. Spectr. Rel. Phen 1, 91 (1977).

${ }^{5}$, R.R. Rye, D.R. Jennison and J.E. Houston, J. Chem. Phys. 10, 4867 (1980).

${ }^{6}$ J.E. Houston, and R.R. Rye, J. Chem. Phys. 1, 71 (1981).

${ }^{7}$ R.R. Rye and J.E. Houston, J. Chem. Phys. 7, 4321 (1983).

${ }^{8}$ Christoph-Maria Liegener, Chemical Physics 1, 97 (1985).

${ }^{9}$ C.-M. Liegener, and E. Weiss, Phys. Rev. B 41, 11946 (1990).

${ }^{10}$ J.H.D.Eland, O. Vieuxmaire, T. Kinugawa, P. Lablanquie, R.I. Hall, and F. Penent,Phys. Rev. Lett. 90, 053003 (2003).

${ }^{11}$ J.H.D Eland, S.S.W Ho and H.L. Worthington, Chem. Phys. 290, 27 (2003).

${ }^{12}$ J.H.D Eland, Chem. Phys. 294, 171 (2003).

${ }^{13}$ J.H.D Eland, R. Feifel, and D. Edvardsson, J. Phys. Chem. A 108, 9721 (2004).

${ }^{14}$ A. Pilcher-Clayton and J.H.D. Eland, J. Electr. Spectr. Rel. Phen. 142, 313 (2005).

${ }^{15}$ R. Feifel, J.H.D. Eland, and D. Edvardsson, J. Chem. Phys. 122, 144308 (2005).

${ }^{16}$ R. Feifel, J.H.D. Eland, L. Storchi, and F. Tarantelli, J. Chem. Phys. 122, 144309 (2005).

${ }^{17}$ R.D. Molloy, A. Danielsson, L. Karlsson and J.H.D. Eland, Chem. Phys. 335, 49 (2007).

${ }^{18}$ P. Linusson, L. Storchi, F. Heijkenskjöld, E. Andersson, M. Elshakre, B. Pfeifer, M. Colombet, J.H.D. Eland, L. Karlsson, J.-E. Rubensson, F. Tarantelli, and R. Feifel, J. Chem. Phys. 129, 234303 (2008).

${ }^{19}$ P. Linusson, L. Hedin, J. H. D. Eland, R. J. Squibb, M. Mucke,S. Zagorodskikh, L. Karlsson, R. Feifel, Phys. Rev. A 88, 022510 (2013).

${ }^{20}$ Y. Hikosaka, T. Aoto, P. Lablanquie, F. Penent, E. Shigemasa, and K. Ito, Phys. Rev. Lett. 97, 053003 (2006).

${ }^{21}$ Y. Hikosaka, T. Kaneyasu, E. Shigemasa, P. Lablanquie, F. Penent, and K. Ito, J. Chem. Phys. 127, 044305 (2007).

${ }^{22}$ E. Andersson, M. Stenrup, J.H.D. Eland, L. Hedin, M. Berglund, L. Karlsson, A Larson, H. Ågren, J.-E. Rubensson, and R. Feifel, Phys. Rev. A 78, 023409 (2008).

${ }^{23}$ E. Andersson, J. Niskanen, L. Hedin, J.H.D. Eland, P. Linusson, L. Karlsson, J.-E. Rubensson, V. Carravetta, H. Ågren, and R. Feifel, J. Chem. Phys. 133, 094305 (2010). 
${ }^{24}$ J. Niskanen, V. Carravetta, O. Vahtras, H. Ågren, H. Aksela, E. Andersson, L. Hedin, P. Linusson, J.H.D. Eland, L. Karlsson, J.-E. Rubensson, and R. Feifel, Phys. Rev. A 82, 043436 (2010).

${ }^{25}$ E. Andersson, P. Linusson, S. Fritzsche, L. Hedin, J.H.D. Eland, L. Karlsson, J.-E. Rubensson, and R. Feifel, Phys. Rev. A 85, 032502 (2012).

${ }^{26}$ J. Niskanen, E. Andersson, J.H.D. Eland, P. Linusson, L. Hedin, L. Karlsson, R. Feifel, and O. Vahtras, Phys. Rev. A 85, 023408 (2012).

${ }^{27}$ J.H.D. Eland, C.F. Rigby, E. Andersson, J. Palaudoux, L. Andric, F. Penent, P. Linusson, L. Hedin, L. Karlsson, J.-E. Rubensson, Y. Hikosaka, K. Ito, P. Lablanquie, and R. Feifel, J. Chem. Phys. 132, 104311 (2010).

${ }^{28}$ J.H.D. Eland, L. Andric, P. Linusson, L. Hedin, S. Plogmaker, J. Palaudoux, F. Penent, P. Lablanquie, and R. Feifel, J. Chem. Phys. 135, 134309 (2011).

${ }^{29}$ F. Penent, J. Palaudoux, P. Lablanquie, L. Andric, R. Feifel, and J.H.D. Eland, Phys. Rev. Lett. 95, 083002 (2005).

${ }^{30}$ J.H.D. Eland, M. Hochlaf, P. Linusson, E. Andersson, L. Hedin, and R. Feifel, J. Chem. Phys. 132, 014311 (2010).

${ }^{31}$ H. Ågren, A. Cesar, and C.M. Liegener, Adv. Quantum. Chem. 23, 1 (1992).

${ }^{32}$ J.H.D. Eland, P. Linusson, M. Mucke and R. Feifel, Chem. Phys. Lett. 548, 90 (2012).

${ }^{33}$ H. Iwayama, N. Sisourat, P. Lablanquie, F. Penent, J. Palaudoux, L. Andric, J.H.D. Eland, K. Bučar, M. Žitnik, Y. Velkov, Y. Hikosaka, M. Nakano, and E. Shigemasa, J. Chem. Phys. 138, 024306 (2013).

${ }^{34}$ D. Chadwick and A. Katrib, J. Electr. Spectr. Rel. Phen. 3, 39 (1974).

${ }^{35}$ W.-C.Tam, D. Yee and C.E. Brion, J. Electr. Spectr. Rel. Phen. 4, 77 (1974).

${ }^{36}$ K. Kimura, S. Katsumata, T. Yamazaki and H. Wakabayashi, J. Electr. Spectr. Rel. Phen. 6, $41(1975)$.

${ }^{37}$ M.P. Keane, S. Lunell, A. Naves de Brito, M. Carlsson-Göthe, S. Svensson, B. Wannberg and L. Karlsson, J. Electr. Spectr. Rel. Phen. 56, 313 (1991).

${ }^{38}$ A.V. Golovin, Yu.L. Sergeev, M.E. Akopyan and F.I. Vilesov, Theoretical and Experimental Chemistry, 13, 580 (1978)

${ }^{39}$ K. Johnson, I. Powis and C.J. Danby, Chem. Phys. 70, 329 (1982).

${ }^{40}$ N. Correia, A. Naves de Brito, M.P. Keane, L. Karlsson, S. Svensson, C.-M. Liegener, A. Cesar, and H. Ågren, J. Chem. Phys. 95, 5187 (1991). 
${ }^{41}$ D. Minelli, F. Tarantelli, A. Sgamellotti and L.S. Cederbaum, J. Electr. Spectr. Rel. Phen. 74, 1 (1995).

${ }^{42}$ P. Kruit and F.H. Read, J. Phys. E: Sci. Instr. 16, 313 (1983).

${ }^{43}$ S. Plogmaker, P. Linusson, J.H.D. Eland, N. Baker, E.M. Johansson, H. Rensmo, R. Feifel, and H. Siegbahn, Rev. Sci. Instr. 83, 013115 (2012).

${ }^{44}$ G.C. King, M. Tronc, F.H. Read and R.C. Bradford, J. Phys. B: At. Mol. Phys. 10, 2479 (1977).

${ }^{45}$ O. Edqvist, E. Lindholm, L.E. Selin, and L. Åsbrink, Physica Scripta 1, 25 (1970).

${ }^{46}$ I.H. Hillier and J. Kendrick, J. Electr. Spectr. Rel. Phen. 6, 325 (1975).

${ }^{47}$ S. Lunell, M. P. Keane and S. Svensson, J. Chem. Phys. 90, 4341 (1989).

${ }^{48}$ J. Colgan, M.S. Pindzola and F. Robicheaux, J. Phys. B: At. Mol. Opt. Phys. 34, L457 (2001).

${ }^{49}$ A. Knapp, A. Kheifets, I. Bray, Th. Weber, A.L. Landers, S. Schössler, T. Jahnke, J. Nickles, S. Kammer, O. Jagutzki, L.Ph.H. Schmidt, T. Osipov, J. Rösch, M.H. Prior, H. Schmidt-Böcking, C.L. Cocke, and R. Dörner, Phys. Rev. Lett. 89, 033004 (2002).

${ }^{50}$ A. Knapp, M. Walter, Th. Weber, A.L. Landers, S. Schössler, T. Jahnke, M. Schöffler, J. Nickles, S. Kammer, O. Jagutzki, L.Ph.H Schmidt, T. Osipov, J. Rösch, M.H. Prior, H. Schmidt-Böcking, C.L. Cocke, J. Feagin, and R. Dörner, J. Phys. B: At. Mol. Opt. Phys. 35, L521 (2002).

${ }^{51}$ J. Colgan, and M.S. Pindzola, Phys. Rev. A 65, 032729 (2002).

${ }^{52}$ T. Schneider, P.L. Chocian, and J.-M. Rost, Phys. Rev. Lett. 89, 073002 (2002).

${ }^{53}$ T. Schneider, and J.-M. Rost, Phys. Rev. A 67, 062704 (2003).

${ }^{54}$ R. Wehlitz, F. Heiser, O. Hemmers, B. Langer, A. Menzel, and U. Becker, Phys. Rev. Lett. 67, 3764 (1991).

${ }^{55}$ S. Svensson et al., J. Electr. Spectr. Rel. Phen. 47, 327 (1988).

${ }^{56}$ Samson et al., Phys. Rev. Lett. 65, 2861 (1990).

${ }^{57}$ S. Zagorodskikh et al., to be published.

${ }^{58}$ J. Hoszowska, A.K. Kheifets, J.-Cl. Dousse, M. Berset, I. Bray, W. Cao, K. Fennane, Y. Kayser, M. Kavčič, J. Szlachetko, and M. Szlachetko, Phys. Rev. Lett. 102, 073006 (2009). 


\begin{tabular}{|c|c|c|c|}
\hline \multicolumn{2}{|c|}{ Carbon, $350 \mathrm{eV}$} & \multicolumn{2}{|c|}{ Oxygen, $600 \mathrm{eV}$} \\
\hline Feature & Assignment & Feature & Assignment \\
\hline \multirow[t]{2}{*}{1} & \multirow[t]{2}{*}{${ }^{1,3} A^{\prime}\left(3 a^{\prime}\right)^{-1}\left(10 a^{\prime}\right)^{-1}$} & \multirow[t]{3}{*}{1} & ${ }^{1,3} A^{\prime}\left(1 a^{\prime}\right)^{-1}\left(10 a^{\prime}\right)^{-1}$ \\
\hline & & & ${ }^{1,3} A^{\prime \prime}\left(1 a^{\prime}\right)^{-1}\left(2 a^{\prime \prime}\right)^{-1}$ \\
\hline 2 & ${ }^{1,3} A^{\prime \prime}\left(3 a^{\prime}\right)^{-1}\left(2 a^{\prime \prime}\right)^{-1}$ & & ${ }^{1,3} A^{\prime}\left(1 a^{\prime}\right)^{-1}\left(9 a^{\prime}\right)^{-1}$ \\
\hline \multirow[t]{2}{*}{3} & ${ }^{1,3} A^{\prime}\left(3 a^{\prime}\right)^{-1}\left(9 a^{\prime}\right)^{-1}$ & \multirow[t]{2}{*}{2} & ${ }^{1,3} A^{\prime}\left(1 a^{\prime}\right)^{-1}\left(8 a^{\prime}\right)^{-1}$ \\
\hline & ${ }^{1,3} A^{\prime}\left(2 a^{\prime}\right)^{-1}\left(10 a^{\prime}\right)^{-1}$ & & ${ }^{3} A^{\prime \prime}\left(1 a^{\prime}\right)^{-1}\left(1 a^{\prime \prime}\right)^{-1}$ \\
\hline \multirow[t]{3}{*}{4} & ${ }^{1,3} A^{\prime \prime}\left(2 a^{\prime}\right)^{-1}\left(2 a^{\prime \prime}\right)^{-1}$ & \multirow[t]{3}{*}{3} & ${ }^{1,3} A^{\prime}\left(1 a^{\prime}\right)^{-1}\left(7 a^{\prime}\right)^{-1}$ \\
\hline & ${ }^{1,3} A^{\prime}\left(3 a^{\prime}\right)^{-1}\left(8 a^{\prime}\right)^{-1}$ & & ${ }^{1} A^{\prime \prime}\left(1 a^{\prime}\right)^{-1}\left(1 a^{\prime \prime}\right)^{-1}$ \\
\hline & ${ }^{1,3} A^{\prime}\left(2 a^{\prime}\right)^{-1}\left(9 a^{\prime}\right)^{-1}$ & & ${ }^{3} A^{\prime}\left(1 a^{\prime}\right)^{-1}\left(6 a^{\prime}\right)^{-1}$ \\
\hline \multirow[t]{3}{*}{5} & ${ }^{1,3} A^{\prime \prime}\left(3 a^{\prime}\right)^{-1}\left(1 a^{\prime \prime}\right)^{-1}$ & & \\
\hline & ${ }^{1,3} A^{\prime}\left(3 a^{\prime}\right)^{-1}\left(7 a^{\prime}\right)^{-1}$ & & \\
\hline & ${ }^{3} A^{\prime \prime}\left(2 a^{\prime}\right)^{-1}\left(1 a^{\prime \prime}\right)^{-1}$ & & \\
\hline \multirow[t]{5}{*}{6} & ${ }^{1,3} A^{\prime \prime}\left(2 a^{\prime}\right)^{-1}\left(1 a^{\prime \prime}\right)^{-1}$ & & \\
\hline & ${ }^{1,3} A^{\prime}\left(2 a^{\prime}\right)^{-1}\left(8 a^{\prime}\right)^{-1}$ & & \\
\hline & ${ }^{1,3} A^{\prime}\left(3 a^{\prime}\right)^{-1}\left(6 a^{\prime}\right)^{-1}$ & & \\
\hline & ${ }^{3} A^{\prime}\left(3 a^{\prime}\right)^{-1}\left(10 a^{\prime} *\right)^{-1}$ & & \\
\hline & ${ }^{3} A^{\prime}\left(2 a^{\prime}\right)^{-1}\left(7 a^{\prime}\right)^{-1}$ & & \\
\hline
\end{tabular}

TABLE IV. Assignment of the core-valence spectra shown in Fig. 8 based on Ref. 57. The left part refers to the spectrum recorded above the Carbon $1 s$ ionization thresholds, and the right part refers to features observed in the spectrum recorded above the oxygen $1 s$ threshold. 